\title{
Die Guten ins Töpfchen ... Auftrag einer forensischen Aufnahmestation (§ 64 StGB)?
}

\author{
Adelheid Bezzel ${ }^{1}$ \\ Eingegangen: 4. Juni 2021 / Angenommen: 9. September 2021 / Online publiziert: 8. Oktober 2021 \\ (c) Der/die Autor(en) 2021
}

\section{Zusammenfassung}

Im Zuge der aktuellen Reformdebatte des § 64 StGB werden Vorschläge zu einer frühen Weichenstellung gemacht, im Sinne einer Selektion durch eine spezialisierte Aufnahmestation. Als Beispiel wird die Regensburger Klinik genannt. Eine Einschätzung der Leistungsfähigkeit des Projektes aus den Jahren 2002/2003 wird im Rahmen der Ergebnisqualitätserfassung MRV (Maßregelvollzug) vorgelegt und legt den Schluss nahe, dass diese Spezialisierung keinen Effekt auf die Qualität der Therapie bzw. des Therapieverlaufes einschließlich des Outcome (Entlassung und Einjahres-Follow-up) hat. Ein negatives Outcome haben $48 \%$ (Entlassjahre 2001-2019) der über 1500 Patienten der Klinik (Entlassung gem. $\S 67 \mathrm{~d}$ Abs. 2, 4, 6 StGB); sie müssen die Therapie vorzeitig verlassen. Einige Risikofaktoren lassen sich insbesondere aus dem kriminologischen Spektrum identifizieren, zudem auch soziodemografische Determinanten wie Alter und Bildung. Weniger problematisch sind die ersten Phasen in der Therapie, vielmehr stellen die Erprobungsphasen am Ende einen risikobehafteten Zeitraum dar: Wer diese erfolgreich besteht, hat große Chancen auf ein straftatfreies Leben (78\%).

Schlüsselwörter Unterbringung in einer Entziehungsanstalt · Forensische Psychotherapie · Erfolgsaussicht ·

Risikofaktoren · Therapieabbruch

\section{The good ones into the potty ... mission of a forensic admission ward ( 64 StGB)?}

\begin{abstract}
Regarding the current reform debate on section 64 of the German Criminal Code (StGB), suggestions are being made for setting the course at an early stage, in the sense of selection by a specialized admission ward. The Regensburg clinic is cited as an example. An evaluation of the project from the years 2002/2003 is presented as part of the outcome quality assessment MRV and suggests that the specialization has no effect on the quality or the course of therapy including the outcome (release and 1-year follow-up). Out of more than 1500 patients (release years 2001-2019) in the clinic (release according to section $67 \mathrm{~d}$ para 2, 4, 6 of the StGB) $48 \%$ have a negative outcome; they have to terminate the therapy early. Risk factors can be identified by criminological and by sociodemographic determinants, such as age and education level. Also, the first phases of therapy are not as problematic as the trial phases at the end of therapy: those who successfully pass the latter have a good chance of a life with impunity $(78 \%)$.
\end{abstract}

Keywords Accomodation in forensic psychiatry $\cdot$ Forensic psychotherapy $\cdot$ Prospect of success $\cdot$ Risk factors $\cdot$ Treatment termination

Dr. phil. Adelheid Bezzel

adelheid.bezzel@medbo.de

$1 \quad$ Klinik für forensische Psychiatrie und Psychotherapie, medbo (KU) Regensburg, IfQM, Katamnese-AG, Regensburg, Deutschland

\section{Einleitung}

Die Unterbringung in einer Entziehungsanstalt nimmt seit Jahren an Bedeutung zu - erfreulich und problematisch zugleich (Müller 2019; Müller et al. 2021; Schalast et al. 2019). Die ansteigenden Belegungszahlen werden zur Herausforderung und provozieren Diskussionen über die „Rich- 
tigen“ im Maßregelvollzug (Berthold und Riedemann 2018, S. 74). Dabei sprach Konrad (1992) bereits von Fehleingewiesenen, machte dies allerdings lediglich an formalen Unterbringungsvoraussetzungen fest. Seifert und Leygraf (1999) gaben dabei zu bedenken, dass diese Einordnung nicht mit der Beurteilung therapeutischer Behandlungsmöglichkeit gleichzusetzen ist; bis heute wird die Problematik der ineffektiven Einweisungshürden beklagt (Querengässer und Baur 2021). Die Kunst, die Richtigen zu filtern, ist für Gutachter eine Herausforderung; viele relevante Faktoren sind unbekannt oder nicht messbar (Berthold und Riedemann 2021). Für die Praxis im MRV folgt ein Dilemma: Auf der einen Seite muss auch der MRV ressourcenschonend arbeiten (d.h. kurze Unterbringung für Fehleingewiesene und Konzentration auf Patienten mit Erfolgsaussicht), auf der anderen Seite ist die Kenntnislage über Risikofaktoren überschaubar, und die Relevanz dynamischer Wirkfaktoren (Verlaufsvariablen, Variablen mit therapeutischer Bearbeitungspotenzial etc.) ist nicht von der Hand zu weisen (Querengässer und Baur 2021). Therapeuten wissen um die Besonderheiten dreier Gruppen: Probanden mit negativen Therapievorerfahrungen, die darauf mit ,resignativem Trotz“ (Schalast 2000, S. 132) reagieren. Darüber hinaus Dissoziale, deren Behandlungsmotivation ebenfalls meist passiv, ablehnend und external ausgerichtet ist (Rauchfleisch 1991). Als dritte Gruppe werden (GUS)Migranten (kulturell unterschiedliche Krankheitskonzepte, Sprachdefizite, Abgrenzung und das sich Zuwenden an eine Subkultur etc.; Bezzel 2009) genannt. Ein Therapieabbruch ist hier nicht zwangsläufig, aber häufig.

Rigoros urteilte Müller (2019) angesichts hoher Abbruchszahlen mit wirtschaftlichen, sicherheitsrelevanten, qualitativen Folgen, dass die Versuche von Gutachter und Gericht zur Erfolgsaussichtsprognose gescheitert seien und daher durch frühzeitige Selektion im MRV gegengesteuert werden müsse. Beispielhaft wird dafür das Projekt spezialisierte Aufnahmestation der Forensischen Klinik Regensburg/Bayern (medbo) in den frühen 2000er-Jahren genannt. Die Intention war eine Identifikation von Therapiegeeigneten innerhalb der ersten Wochen bzw. die Selektion anderer (Erledigung). Erwartet wurden positive Auswirkungen auf Therapiequalität, -ergebnisse sowie Belegungssteuerung; eine Effektivitätsmessung erfolgte anhand einer einjährigen Aufnahmekohorten(AK)-Beobachtung (Müller 2005). Aufgrund des kurzen Zeitraumes war es allerdings nicht möglich, den weiteren Verlauf der auf der Aufnahmestation als geeignet befundenen Patienten zu verfolgen. Dieses Forschungsdesiderat wird nun aufgenommen.

\section{Ergebnisse}

\section{Methodeneinführung und Forschungsinteresse}

Müller (2005) untersuchte in seiner Outcome-Analyse eine AK (01.11.2002-31.10.2003; $n_{\mathrm{AK}}=152$ Patienten), berücksichtigte dabei aber keine Störvariablen: Nichterfassen der Erledigungen zu einem späteren Unterbringungszeitpunkt, keine Bereinigung der Stichprobengrundgesamtheit um Patienten mit sonstigen (organisatorischen, strukturellen) Entlassgründen (z. B. Entlassung in den Zwischenvollzug, Verlegung etc.) und Einbeziehung der vorläufig Untergebrachten ( $\$ 126 \mathrm{a}$ StPO), die aufgrund der ausstehenden Eingangsmerkmalprüfung bzw. abweichenden Therapierahmenbedingungen für MRV-Outcome-Analysen ungeeignet sind. Zudem beschränkte er sich auf eine einjährige Beobachtungszeit. Die aktuelle Analyse dagegen kann Gesamtverläufe der Entlassjahre (EJ) 2001-2019 untersuchen und damit auch Patienten aus der AK bis zur realen Entlassung erfassen; Grundlage ist die seit 2001 in der Klinik als Bestandteil des QM etablierte Ergebnisqualitätserfassung (u. a. Bezzel 2008). Alle Patienten (Vollerhebung) werden nach mindestens 6-wöchiger Unterbringung zum Zeitpunkt der Entlassung ( $\$ 67 \mathrm{~d}$ Abs. 2, 4, $\left.6 \mathrm{StGB} ; N_{\mathrm{GSP}}=1755\right)$ untersucht; bei Probandeneinverständnis wird sowohl eine $\mathrm{Zu}$ friedenheitsbewertung wie auch nach einjähriger Bewährungszeit eine Katamnese angeschlossen, mit Nutzung von Probanden- und Fremdangaben (z. B. Bewährungshilfe).

Ausgehend von der damaligen Projektbewertung der spezialisierten Aufnahmestation werden die Verläufe und Outcome-Entwicklungen erneut untersucht und mit den Daten aus 2 Jahrzehnten in Beziehung gesetzt. Dabei werden folgende Fragestellungen beleuchtet: Veränderung von Erledigerraten, Extraktion sensibler Therapiephasen und von Risikomerkmalen, Ausflüsse auf Qualitätsmerkmale durch frühzeitige Selektion.

\section{Erledigungen im Gesamtverlauf und die zeitliche Entwicklung}

Müller (2005) berechnete in seiner AK eine 33\%ige Erledigerrate in der Einjahresbeobachtung. Um die Frage zu klären, ob möglichst alle „Fehleingewiesenen“ frühzeitig identifiziert werden konnten, werden die Kohortenpatienten im Gesamtverlauf analysiert: Die tatsächliche Erledigerrate liegt dann bei $48,7 \%$.

Vorläufig Untergebrachte haben eine auffallend hohe Abbruchsquote (Tab. 1). Schließt man, um die tatsächliche Leistungsfähigkeit des MRV einschätzen zu können, diese Sondergruppe und Patienten mit sonstigen Entlassgründen aus und berechnet die Entlassartverteilung aller Regensburger §-64-StGB-Patienten der vergangenen 2 Jahrzehnte $\left(n_{\mathrm{GSP}}=1755\right)$, liegt die Erledigerquote bei $47,9 \%$. 
Tab. 1 Entlassungen im Gesamtverlauf (Aufnahmekohorte, $n_{\mathrm{AK}}=154$ )

\begin{tabular}{lll}
\hline & $\begin{array}{l}\S 126 \mathrm{a} \text { StPO } \\
n_{126 \mathrm{a}}=22\end{array}$ & $\begin{array}{l}\S 64 \text { StGB } \\
n_{64}=132\end{array}$ \\
\hline Bedingte Entlassung & $1(4,5 \%)$ & $39(29,5 \%)$ \\
Abbruch wegen Aussichtslosigkeit/ & $15(68,2 \%)$ & $60(45,5 \%)$ \\
$\begin{array}{l}\text { Erledigung } \\
\text { Höchstfrist }\end{array}$ & - & $3(2,3 \%)$ \\
$\begin{array}{l}\text { Sonst: Rückstellung §35 BtMG, } \\
\text { Verlegung in andere Einrichtung, } \\
\text { verstorben, Zwischenvollzug, Ent- } \\
\text { weichung (formale Entlassung) etc. }\end{array}$ & $6(27,3 \%)$ & $30(22,7 \%)$ \\
\hline
\end{tabular}

Es besteht ein zeitlicher Zusammenhang $\left(K^{+}=0,712\right.$; $p<0,05)$, die Erledigerquote nimmt - nach einem Anstieg im Zuge des Aufnahmestationsprojektes (s. unten) tendenziell in den letzten Jahren ab. Auffällig ist ein Wendepunkt ab 2013: Die Positiv-Outcome-Rate überwiegt $(60,5 \%$ vs. $42,3 \%$ vor 2013 , Exakter Test nach Fisher $=65,066, p<0,05)$. Quantitative Risikomerkmalsverschiebungen, aber auch konzeptionelle Änderungen werden als sekundierend angenommen. In der jüngeren Vergangenheit hat sich der Umgang mit sog. schwierigen Patienten geändert; es wurden in Regensburg andere therapeutische Angebote eingeführt. Bis 2013 z.B. wurden nur 6 Probanden mit entsprechender Diagnose substituiert, danach steigert sich die Quote auf $31 \%$ (Exakter Test nach Fisher $=155,953, p<0,05$ ).

\section{Sensible Therapiephasen}

Da der Entlasszeitpunkt nicht den wahren Therapieabbruchszeitpunkt wiedergibt und u. a. von der Verschubungspraxis abhängig ist, wird ab 2006 der Abbruchsantragszeitpunkt erhoben ( $n=577)$. Im Schnitt stellen die Therapeuten ggf. nach 13 Monaten einen Antrag auf Erledigung (min: OMon., max: 51 Mon., Median =11,00, $\pm 8,611$ ). Fragt man nach der erreichten Lockerungsstufe bei Antragsstellung, können 2 Phasen als besonders risikoreich separiert werden: der Therapiebeginn (16,8\%) und v.a. die Phase mit dem höchsten Lockerungsgrad gegen Ende der konzipierten Therapie (40,2\% aller Abbrüche). Die meisten - zunächst nicht als sog. Fehleingewiesene deklariert - werden lange therapiert und begleitet, um dann doch zu scheitern (Ø 351,39 Tage, min: 26T., max: 1414T., Median=196, $\pm 368,570$ ). Auch in der Projektevaluation der AK hätte sich dies bei Beachtung des Gesamtverlaufes gezeigt: Bei einjähriger Beobachtung gelten $80 \%$ als Frühidentifizierte (Müller 2005), in der Gesamtschau aber nur knapp $20 \%$ der AK (innerhalb der ersten 90 Tage abgebrochen und entlassen).

Späte Erledigungen verursachen inhaltslogisch höhere Unterbringungsdauern. Wie die Gesamtschau der letzten 20 Jahre zeigt $(F(18)=5,254, p<0,05$; Abb. 1), nehmen die Therapiedauern bei Erledigungen in Regensburg tendenziell zu. Ein Hinweis, dass sich der Versuch, frühzeitig
Abb. 1 Mittlere Unterbringungsdauer bei Erledigungen $(n=840)$, EJ 2001-2019 (Regensburg)

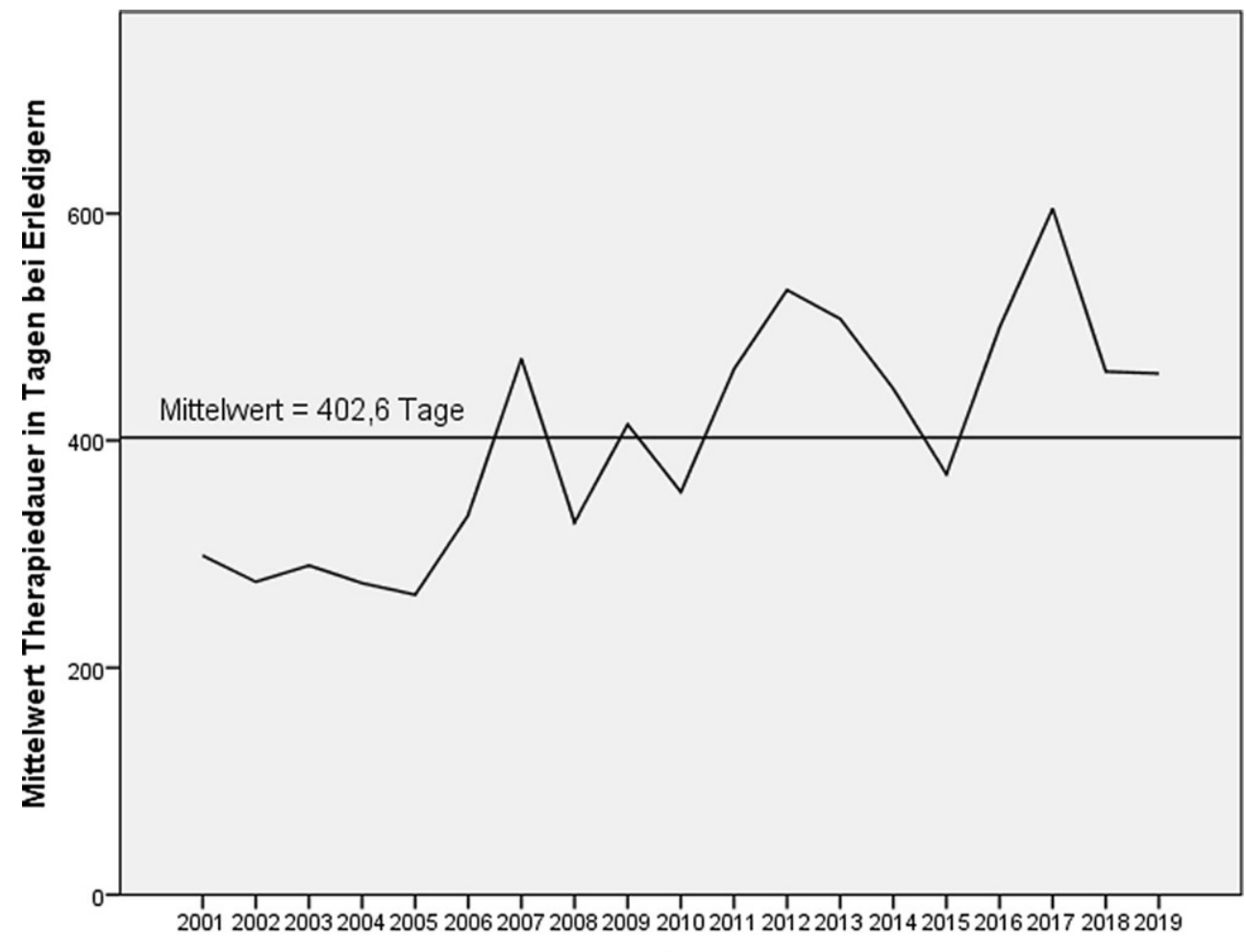

Jahr der Entlassung 
zu selektieren, nicht durchgesetzt und sich die Praxis ab 2006 geändert hat.

\section{Personale Risikomerkmale}

Indikatoren, die für oder gegen eine erfolgreiche Therapie im Sinne eines positiven Outcome sprechen, sind nicht nur für die Prognose im Erkenntnisverfahren relevant, sondern spielen auch während der Unterbringung eine Rolle. Die Befundlage ist aber heterogen, zudem sind Einflüsse der Klinik, des Settings, der Justiz nicht auszuschließen, wenn nicht sogar besonders bedeutsam (eine Übersicht bei Querengässer und Baur 2021). Die in Regensburger Studien als relevant erachteten Risikofaktoren (Bezzel 2009; Hartl 2012) lassen sich auch in der aktuellen Analyse replizieren (Tab. 2). Das kriminologische Spektrum scheint von besonderer Brisanz (kommensurabel bei Querengässer und Baur 2021). Auch die schulische Qualifikation hat Einfluss $\left(K^{+}=0,188 ; p<0.05\right)$ : Patienten mit maximal einfachem Hauptschulabschluss haben höhere Erledigerquoten (bis zu zwei Drittel). Andere Items (Ersttat, Geschlecht,

Tab. 2 Risikomerkmale, EJ 2001-2019 (Regensburg)

\begin{tabular}{|c|c|c|}
\hline $\begin{array}{l}\text { Bedingt Entlassene } \\
(n=865)\end{array}$ & $\begin{array}{l}\text { Merkmal aus der Vorge- } \\
\text { schichte bzw. zu Thera- } \\
\text { piebeginn }\end{array}$ & $\begin{array}{l}\text { Erlediger } \\
(n=842)\end{array}$ \\
\hline $28,1 \%(n=241)$ & $\begin{array}{l}\text { Diagnose: Polytoxiko- } \\
\text { manie } \\
\left(K^{+}=0,230 ; p<0,05\right)\end{array}$ & $\begin{array}{l}40,8 \% \\
(n=342)\end{array}$ \\
\hline $\begin{array}{l}12,6 \%(n=109) \\
55,4 \%(n=479)\end{array}$ & $\begin{array}{l}\text { Anlassdelikt: } \\
\text { Eigentumsdelikt } \\
\text { BtMG } \\
\left(K^{+}=0,235 ; p<0,05\right)\end{array}$ & $\begin{array}{l}24,8 \% \\
(n=208) \\
33,1 \% \\
(n=277)\end{array}$ \\
\hline $32,29 \mathrm{~J}$ & $\begin{array}{l}\text { Aufnahmealter } \\
(t(1702)=3,341 ; p<0,05)\end{array}$ & $30,95 \mathrm{~J}$ \\
\hline 38,78 Mon & $\begin{array}{l}\text { Höhe der FHS } \\
(t(1679,449)=11,385 \\
p<0,05)\end{array}$ & 27,95 Mon \\
\hline 8,63 Mon & $\begin{array}{l}\text { Haftzeit vor MRV } \\
(t(1690)=3,973 ; p<0,05)\end{array}$ & 6,66 Mon \\
\hline 30,77 Mon & $\begin{array}{l}\text { Hafterfahrung } \\
(t(1630,790)=-3,591 \\
p<0,05)\end{array}$ & 37,29 Mon \\
\hline 7,34 & $\begin{array}{l}\text { BZR } \\
(t(1633)=-5,839 \\
p<0,05)\end{array}$ & 8,91 \\
\hline $20,48 \mathrm{~J}$ & $\begin{array}{l}\text { Alter bei } 1 \text {. Verurteilung } \\
(t(834,393)=0,223 \\
p<0,05)\end{array}$ & $18,58 \mathrm{~J}$ \\
\hline 0,20 & $\begin{array}{l}\text { Vorbehandlungen im } \\
\text { MRV } \\
(t(1578,542)=-6,107 \\
p<0,05)\end{array}$ & 0,35 \\
\hline $13,85 \mathrm{~J} .(n=272)$ & $\begin{array}{l}\text { Migration: wie viele } \\
\text { Jahre in Deutschland? } \\
(t(506,044)=5,623 ; \\
p<0,05)\end{array}$ & $\begin{array}{l}9,9 \mathrm{~J} . \\
(n=288)\end{array}$ \\
\hline
\end{tabular}

Belastungserleben, Ersttatalter etc.) sind als Diskriminationsmerkmale nicht geeignet (n.s. bzw. mit sehr kleinem Effekt).

Bezüglich der Therapiezeit spielen erwartungsgemäß Entweichungen eine Rolle $(t(1067,845)=-10,817 ; p<0,05$ : 0,03 vs. 0,22 ), $19,2 \%$ der Erlediger sind Flüchtige (vs. $\left.2,7 \% ; K^{+}=0,257 ; p<0,05\right)$. Auch Suchtmittelrückfälle sind relevante Risikoereignisse: $48,3 \%$ (vs. $32,7 \%$ ) konsumieren mind. einmal $\left(K^{+}=0,356 ; p<0,05\right)$. Häufen sich die Rückfälle, steigt das Abbruchsrisiko (1,94 vs. 4,79; $t(1073,321)=-6,137 ; p<0,05)$. Bei 67,2\% der Gescheiterten (vs. $40 \%$ ) werden besondere Behandlungsprobleme mit Motivation, Absprachefähigkeit und ausländerrechtlichem Status etc. von den Behandlern beschrieben $\left(K^{+}=0,268\right.$; $p<0,05$ ). Protektiv dagegen wirkt eine Partnerschaft (Exakter Test nach Fisher $=188,655 ; p<0,05: 74,7 \%$ vs. $25,3 \%$ ). In der Ergebnisqualitätserfassung werden ab 2006 auch die Angaben der Therapeuten zu den im Abbruchsantrag angegebenen Gründen erhoben: Fortwährender Substanzmissbrauch $(47,7 \%)$ und defizitäre Therapiemotivation $(46,6 \%)$ sind dabei die häufigsten. Weniger prominent sind disziplinarische Gründe bzw. Gewalt $(<15 \%)$. Etwa zwei Drittel der Betroffenen sind nicht mit dem Therapieabbruch einverstanden, ein Viertel dagegen regt den Abbruch selbst an.

Es ist nicht publiziert, welche Merkmale 2002/2003 bei Patienten der spezialisierten Aufnahmestation als entscheidungsrelevant gesehen wurden. Die Klientel scheint auch nicht übermäßig stark belastet gewesen zu sein; die üblichen Risikofaktoren sind bis auf 3 Ausnahmen lediglich in geringem Ausmaß vertreten (Tab. 3). Befragt man die damaligen Therapeuten, werden Suchtmittelrückfälle, unklare Abstinenzmotivation, disziplinarische Schwierigkeiten sowie strukturelle Probleme (z.B. ausländerrechtlicher Status, Sprache) als damalig bedeutsam erachtete Therapiehindernisse genannt.

Tab. 3 Risikomerkmale, nur Aufnahmekohorte (zwischen 01.11.2002-31.10.2003)

\begin{tabular}{|c|c|c|}
\hline $\begin{array}{l}\text { Bedingt Entlassene } \\
(n=40)\end{array}$ & Merkmal & $\begin{array}{l}\text { Erlediger } \\
(n=61)\end{array}$ \\
\hline $35,20 \mathrm{~J}$ & $\begin{array}{l}\text { Aufnahmealter } \\
(t(83,471)=2,263 ; p<0,05)\end{array}$ & $30,95 \mathrm{~J}$ \\
\hline 32,98 Mon & $\begin{array}{l}\text { Höhe FHS } \\
(t(86,501)=2,154 ; p<0,05)\end{array}$ & 25,92 Mon \\
\hline $68,4 \%(n=13)$ & $\begin{array}{l}\text { Partnerschaft } \\
\text { (Exakter Test nach Fish- } \\
\text { er }=8,491 ; p<0,05)\end{array}$ & $31,6 \%(n=6)$ \\
\hline
\end{tabular}




\section{Keine Qualitätssteigerung durch frühzeitige Selektion}

In $46,1 \%$ aller Entlassfälle ( $\left.n_{\mathrm{GSP}}=1757\right)$ sehen die Therapeuten rückblickend keine besonderen Behandlungsprobleme - separiert nach Entlassmodus erwartungsgemäß deutlich zuungunsten der Erlediger; d.h. zum einen, Zwischenfälle wie Lockerungsmissbrauch und Suchtmittelrückfall sind insgesamt weniger häufig als angenommen, zum anderen scheint die Überlegung, dass frühzeitige Selektion die Zwischenfallproblematik im weiteren Verlauf entschärft, theoretisch richtig zu sein. Allerdings kann die längsschnittliche Analyse dies nicht bekräftigen; die Quoten unerwünschter Ereignisse unterscheiden sich jahrgangsbezogen nicht, somit auch nicht im Zuge der Einführung der Projektstation. Auch die Therapeuteneinschätzungen sind in allen Auswertungsjahrgängen ähnlich: Über die Hälfte der Patienten sei in Behandlung und Umgang schwierig gewesen - unabhängig davon, ob aus der spezialisierten Aufnahmestation kommend oder nicht.

Eine positive Auswirkung der Frühselektion auf das Therapieklima wurde damals ebenfalls angenommen. Einen Hinweis auf atmosphärische Aspekte geben Feedbacks der regulär entlassenen Patienten am Therapieende $\left(n_{\text {Interview }}=748 ;\right.$, ,missing“: $\left.13,6 \%\right)$. Die Patienten aus der Aufnahmekohorte unterscheiden sich in ihrem Erleben aber kaum von anderen Jahrgängen. Im Gegenteil, wird explizit nach der Stationsatmosphäre (Wohlbefinden, ge- schützter Rahmen, Offenheit, Wertschätzung etc.) gefragt, schildern Jahrgänge jüngerer Zeit diese positiver als andere $\left(K^{+}=0,268 ; p<0,05\right)$. Benotungen (Schulnoten) fallen zwar unterschiedlich aus $\left(K^{+}=0,386 ; p<0,05\right)$, jedoch weder in der Klarheit, wie u.U. im Zusammenhang mit einer frühen Auslesepraxis erwartet, noch mit besseren Noten durch Projektpatienten. Insgesamt sind 15,5\% der bedingt Entlassenen der Gesamtstichprobe sehr zufrieden, mit der Unterbringung bzw. therapeutischen Bausteinen, 46,3\% geben die Note 2, 28,5\% die Note 3 .

\section{Therapieerfolg (Outcomes I und II)}

Therapieerfolg ist in 2 Stufen gliederbar (Querengässer und Baur 2021): Erreichen eines regulären Therapieabschlusses (bedingte Entlassung nach vollzogener Therapie) sowie katamnestisch ermittelter Langzeiterfolg nach der Unterbringung. $\mathrm{Zu}$ Outcome I gibt es eine Jahrgangsverschiebung (Abb. $2 ; K^{+}=0,209 ; p<0,05$ ). Stellt man die Aufnahmekohorte 2002/2003 in Bezug zur Reststichprobe, zeigt sich ein Unterschied (aber n. s.): Nur ein gutes Drittel kann letztlich bedingt entlassen werden (vs. die Hälfte der Reststichprobe); erwartbar im Zusammenhang mit dem Projektauftrag, aber nicht erklärbar, z.B. durch eine etwaige Kumulation der als riskant bekannten Faktoren dieser Jahrgänge.

Für die Effektivitätsbemessung der Maßnahme an sich relevanter ist allerdings die Messgröße Therapieerfolg nach einem Jahr (Outcome II). Dazu werden erfolgreich zu En-
Abb. 2 Aufnahmejahrgänge und späterer Therapieabschluss, 1999-2018 (Regensburg)

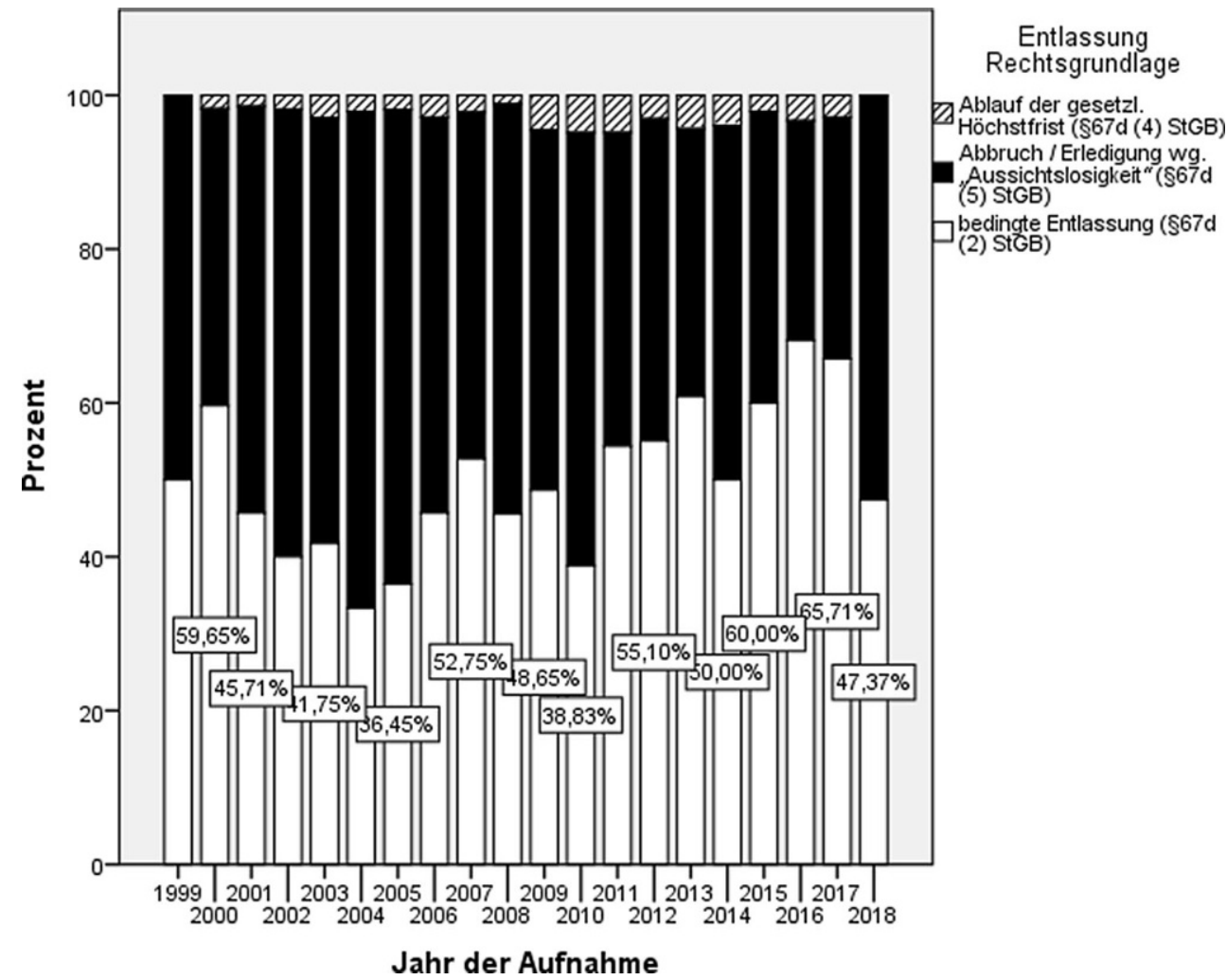


Abb. 3 Aufnahmejahrgang und Sucht (Katamnese nach einem Jahr), bedingt Entlassene 1999-2018 (Regensburg)

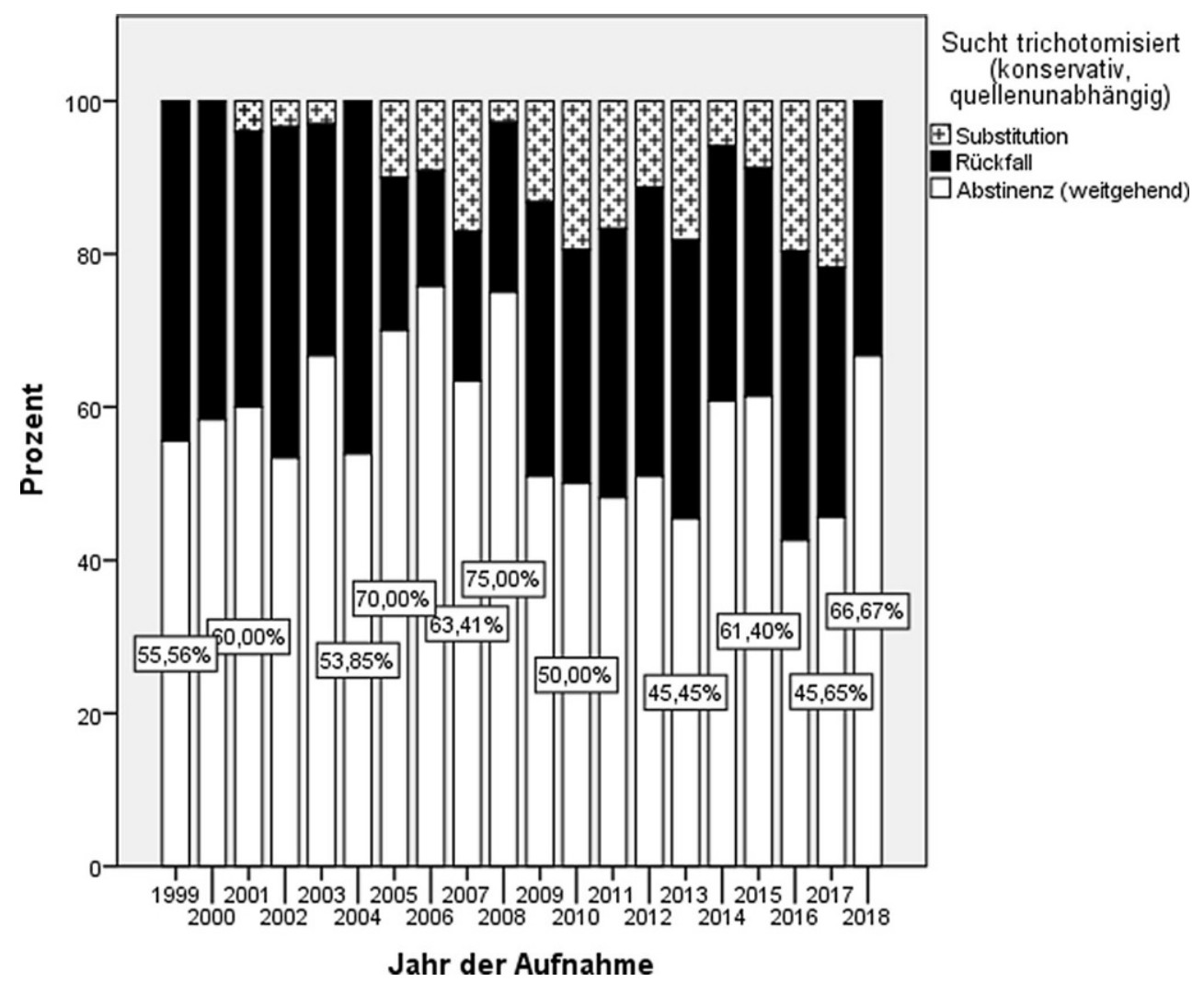

de Therapierte nach einem Jahr in Freiheit interviewt bzw. Fremdinformationen von der Bewährungshilfe etc. eingeholt (Bezzel und Schlögl 2021) - mit Nutzung zweier Quellen kann Antwortverzerrungen begegnet und zudem der Informationsgehalt erhöht werden, dennoch sind Selektionseffekte aufgrund des Einverständnisvorbehaltes nicht auszuschließen. Von 766 Regensburger katamnestisch erfassten Probanden leben $51 \%$ im ersten poststationären Jahr abstinent und straftatfrei (def. Legalbewährung: weder selbstnoch fremdberichtete delinquente Verhaltensweisen unabhängig von strafrechtlicher Sanktion bzw. behördlicher Bekanntheit). Weitere $27,8 \%$ konsumieren in unterschiedlicher Qualität und Quantität, bleiben aber ebenfalls legalbewährt. Die Aufnahmekohorte 2002/2003, die nach ihrer Konzeption entsprechend viele als Fehleingewiesene definiert und selektiert hat, schneidet nicht signifikant besser $\mathrm{ab}$ - weder in der Gesamtbewertung, noch in der differenzierteren Erfolgsbewertung nach Sucht und Legalverhalten.

Splittet man die beiden Erfolgsparameter, bleiben 56,2\% der Gesamtstichprobe weitgehend oder stabil abstinent, $11,1 \%$ werden zum Zeitpunkt der Erhebung substituiert (mit/ohne Beikonsum), und 32,6\% konsumieren mehr oder weniger dauerhaft. In Abhängigkeit vom Aufnahmejahrgang sind Verschiebungen in den 3 Suchtkategorien zu sehen $\left(K^{+}=0,269 ; p<0,05\right.$; Abb. 3), jedoch ohne Sonderstellung der Aufnahmekohorte (2002/2003: 53,3/66,7\% Abstinenzquote).
Hinsichtlich des zweiten Erfolgsparameters (Legalbewährung) ist ebenfalls kein Jahrgang besonders herausstechend, auch nicht Patienten aus der ehemaligen Projektstation: Insgesamt leben $77,9 \%$ ohne strafrechtlich relevante Verhaltensweisen. Es lassen sich keine Hinweise darauf finden, dass eine hohe Selektionsquote eine Steigerung des positiven Outcome der anderen zur Folge hat.

\section{Diskussion}

Eine Therapie nur denjenigen Patienten anzubieten, die davon profitieren können (und am besten auch wollen), ist ein nachvollziehbarer und legitimer Anspruch, der auch in den Eingangsvoraussetzungen $\mathrm{zu}$ finden ist (positive $\mathrm{Be}$ handlungserfolgsprognose) - aber eine evtl. allzu komplexe Aufgabe für die Begutachtung (Querengässer und Baur 2021)? Wenn massive Überbelegung droht, nimmt die Klage über potenziell Fehleingewiesene zu. Abbruchquoten werden nicht als Hinweis auf Behandlungsdefizite, sondern als Beleg für die falsche Einweisungspraxis angeführt (Müller 2019); eine durchaus kritisch zu hinterfragende Schlussfolgerung angesichts großer Schwankungsbreite der Datenlage (30-70\%) und nachgewiesener Klinik- bzw. Behandlungseinflüsse (Berthold und Riedemann 2021; Pollähne und Kemper 2007; Schalast 2021). Zweifelsohne gibt es Patienten, die nicht erreichbar sind; diese frühzeitig zu 
identifizieren, könnte in vielerlei Hinsicht sinnvoll sein und war Grundidee des Regensburger Aufnahmestationsprojektes. Der erwünschte Effekt trat nicht in erwartetem Ausmaß ein; de facto wurde das Experiment nach wenigen Jahren aufgegeben. Fragt man beteiligte Therapeuten $\mathrm{zu}$ den Gründen, geben sie die Unschärfe der Selektionsvariablen (bestätigt durch die vorliegende Gesamtverlaufsanalyse, da nur ein Teil der letztlich Gescheiterten frühzeitig erkannt wurde), ihre Überforderung mit der geforderten Geschwindigkeit der Abbruchsentscheidungen und eine angespannte Stationsatmosphäre an. Die Kollegen der Therapiestationen wiederum hätten keinen Effekt auf das Therapieklima, die Behandlungsmöglichkeiten und das Outcome feststellen können. Zudem spielte ein anderer Partner nicht in dem Maße mit, wie es für eine spürbare Entlastung der Situation nötig gewesen wäre: Die entscheidende Strafvollstreckungskammer, welche die forcierte Geschwindigkeit problematisierte und als nachträgliche Korrektur des erkennenden Gerichtsurteils kritisierte. Eine mindestens halbjährige Unterbringung wurde gefordert. Das Ziel der Kürzung der Unterbringungszeiten war zudem nicht haltbar, da häufig und insbesondere bei fehlender Zustimmung zum Abbruch seitens der Patienten zwischen Antrag und tatsächlicher Verschubung viele Wochen lagen.

Das Projekt in Regensburg offenbart die Schwierigkeiten in der Erfassung von sog. Fehleingewiesenen. Mittlerweile liegen einige Ergebnisse zu Risikofaktoren vor; auch die Regensburger Analyse verweist darauf (u.a. Berthold und Riedemann 2021): kriminelle Vorbelastung, problematischer sozialer Hintergrund und Bildungshistorie. Diese sind aber nicht hinreichend, um die hohe Erledigerquote in der Projektphase zu erklären. Befragt man die damaligen Therapeuten, werden Merkmale als damals entscheidend genannt, die eher an die Führbarkeit oder schlicht das gute Benehmen bzw. die soziale Kompetenz denken lassen: Ältere Patienten, anpassungsfähige BtMGler, Sprachgewandte mit ausreichender Beschulung bzw. beruflicher Qualifizierung, Patienten ohne ausgeprägte Haftsozialisation, Frauen. $\mathrm{Ob}$ diese wirklich sinnvolle Entscheidungskriterien für die Einschätzung eines Therapieerfolges sind, bezweifeln heute selbst die Therapeuten: Gerade diejenigen, die anfangs sehr überzeugten, hätten die nötige Ausdauer vermissen lassen und mussten (später) die Therapie verlassen. Vielleicht wäre ein angepasstes Behandlungsprogramm effektiv gewesen, oder die Unterbringung war zu kurz (Berthold und Riedemann 2021). Ein Arbeitsbündnis sollte nach Rauchfleisch (1999) nicht Voraussetzung, sondern zeitintensives Teilziel sein - eine Aufgabe für Patient und Therapeut (Bachelor 2013) unter Beachtung der Ausgangslage und Einflussfaktoren (s. a. epistemisches Vertrauen und dessen Kontextabhängigkeit; Wilson und Sperber 2012). Therapeuten gehen von einem langen Atem in der Therapie aus, da gerade motivationale Aspekte vielschichtig, wandelbar und zu- mindest indirekt auch von externen Faktoren abhängig sind, was sie als (dichotome) Eingangsvoraussetzungen ungeeignet macht (Schalast 2000; Querengässer et al. 2014). Manche Effekte zeigen sich auch erst nach dem MRV. Die Regensburger sog. Abbrecherkatamnese (ein Jahr nach Haftentlassung; Bezzel 2020) beschreibt, dass einige gescheiterte Patienten (oft mit Erledigungen in späteren Therapiephasen) durchaus in der Lage sind, straftatfrei zu leben trotz schlechterer Ausgangslage aufgrund der Haft. Die Abbrecherkatamnesen offenbaren zudem die Verzahnung von sozialer Integration, Konsum- und Legalverhalten und unterstreichen die Bedeutung der forensischen Resozialisierungsphase mit entsprechend langer Dauer.

Eine Aufnahmestation mit der Aufgabe, die Richtigen ins Töpfchen, die Falschen ins Kröpfchen zu separieren, ist überfordert: Es kann allenfalls nur ein Teil der letztlich erledigten Patienten als problematisch identifiziert werden, unklare Kriterien begünstigen u.U. eine Benachteiligung von (unangenehmen) Patienten; Arbeits- und Therapieklima werden beeinträchtigt. Die Annahme, die Weiterbehandelten hätten durch Selektion zu einem frühen Therapiezeitpunkt Vorteile, wenn frühzeitig abgebrochen wird, kann weder mit quantitativen noch qualitativen Daten unterfüttert werden. Die Chancen auf ein positives Outcome scheinen dadurch nicht verbessert: Unabhängig vom Jahrgang liegen die Therapieerfolge nach einem Jahr in einem zufriedenstellenden Bereich; d.h., auch wenn viele Patienten möglichst rasch abgebrochen werden, steigen die Therapieerfolgsquoten anschließend nicht.

Andere Bausteine zur Steigerung des Erfolges sind besser geeignet, wie die Zahlen aus 20 Jahren Ergebnisqualitätserfassung in Regensburg zeigen. Effektiv sind u.a., wie auch Berthold und Riedemann (2021) aus einer bundesweiten Risikomerkmalanalyse resümieren, ausreichend Zeit, Aufmerksamkeit für Patienten mit Risikofaktoren (z. B. jugendlicher Delinquenzbeginn, negative Therapieerfahrung), Angebote von Substitution und Bildung. Von Vorteil sind Konzepte, welche die Vielschichtigkeit von Therapiewillen, -motivation, -fähigkeit beachten. Beispielhaft sei die mentalisierungsbasierte Therapie genannt; auch die Beachtung des sozialen Umfelds (Querengässer et al. 2014) und insbesondere die Arbeit an Resozialisierungsbausteinen (Bezzel 2020) sind erfolgsversprechend. Mitarbeitende tun gut daran, einen Perspektivenwandel zu versuchen: Realistischere Zielsetzungen mit Bessern statt Heilen (Berthold und Riedemann 2021; Schalast 2019) bzw. Aufgabe des „Dogmas der völligen Abstinenz“, wie es Mache als ehemaliger Chefarzt der Klinik in seinem Grußwort zum 10jährigen Bestehen des IfQM (2021) formuliert. Weiter ermuntert er dazu, die Forderung nach Willensstärke aufzugeben; er empfiehlt eine andere Sicht: ,Vielleicht sollten wir es ernst nehmen und die Patienten während eines Teils ihres Lebens ,be- 
gleiten " und nicht deren Leben als moralisch übergeordnete Person bestimmen wollen.“

Funding Open Access funding enabled and organized by Projekt DEAL.

Interessenkonflikt A. Bezzel gibt an, dass kein Interessenkonflikt besteht.

Open Access Dieser Artikel wird unter der Creative Commons Namensnennung 4.0 International Lizenz veröffentlicht, welche die Nutzung, Vervielfältigung, Bearbeitung, Verbreitung und Wiedergabe in jeglichem Medium und Format erlaubt, sofern Sie den/die ursprünglichen Autor(en) und die Quelle ordnungsgemäß nennen, einen Link zur Creative Commons Lizenz beifügen und angeben, ob Änderungen vorgenommen wurden.

Die in diesem Artikel enthaltenen Bilder und sonstiges Drittmaterial unterliegen ebenfalls der genannten Creative Commons Lizenz, sofern sich aus der Abbildungslegende nichts anderes ergibt. Sofern das betreffende Material nicht unter der genannten Creative Commons Lizenz steht und die betreffende Handlung nicht nach gesetzlichen Vorschriften erlaubt ist, ist für die oben aufgeführten Weiterverwendungen des Materials die Einwilligung des jeweiligen Rechteinhabers einzuholen.

Weitere Details zur Lizenz entnehmen Sie bitte der Lizenzinformation auf http://creativecommons.org/licenses/by/4.0/deed.de.

\section{Literatur}

Bachelor A (2013) Clients' and therapists' views of the therapeutic alliance: similarities, differences and relationship to therapy outcome. Clin Psychol Psychother 20:118-135

Berthold D, Riedemann C (2018) Behandeln wir eigentlich (noch) die Richtigen? Eine retrospektive Betrachtung der Unterbringung gemäß §64 StGB. Forens Psychiatr Psychother 25:74-90

Berthold D, Riedemann C (2021) Welche Patienten haben eine ,hinreichend konkrete Aussicht" auf einen Behandlungserfolg in der Maßregel nach § 64 StGB? Forens Psychiatr Psychol Kriminol. https://doi.org/10.1007/s11757-021-00653-0

Bezzel A (2008) Therapie im Maßregelvollzug - und dann? Eine Verlaufsuntersuchung an forensischen Patienten ( $\$ \S 63$ und 64 StGB). Dissertation, Universität Regensburg. http://www.opusbayern.de/uni-regensburg/volltexte/2009/1096/

Bezzel A (2009) Therapieabbruch im Maßregelvollzug ( $\$ 64$ StGB) - Charakteristika und Prädiktoren. Prax Rechtspsychol 19(1): $146-153$

Bezzel A (2020) Der MRV als Brückenbauer - aus der Ergebnisqualitätsmessung Bayern. In: Müller J, Koller M (Hrsg) Reformansätze zur Unterbringung nach $§ 64$ StGB. Kohlhammer, Stuttgart, S 42-56
Bezzel A, Schlögl C (2021) Eine Retrospektive zum bayerischen Maßregelvollzug: ,alles bewegt sich fort und nichts bleibt“"? Forens Psychiatr Psychother 29(2):151-172

Hartl C (2012) Wie erfolgreich ist die Behandlung im Maßregelvollzug nach $\S \S 63$ und 64 StGB? Dissertation, Universität Regensburg

Konrad N (1992) Zur Beachtung der Einweisungskriterien bei Unterbringungen in einer Entziehungsanstalt gemäß $\S 64$ StGB. Strafverteidiger 12:597-602

Müller J (2005) Motivation oder Aussichtslosigkeit? Konzept und 1-Jahres-Evaluation einer suchtforensischen Aufnahme-, Diagnostik, und Motivationsstation. Psychiatr Prax 32(4):205-207

Müller J (2019) Ansätze zur Reform der Unterbringung in einer Entziehungsanstalt. Forens Psychiatr Psychol Kriminol. https://doi. org/10.1007/s11757-019-00542-7

Müller J, Böcker F, Eusterschulte B, Koller M, Muysers J, Pollmächer T (2021) Neuregelung des $\S 64$ StGB aus psychiatrischer Sicht. Positionspapier einer Task-Force der DGPPN. Nervenarzt. https://doi.org/10.1007/s00115-021-01109-w

Pollähne H, Kemper A (2007) Fehleinweisungen in die Entziehungsanstalt ( $\$ 64 \mathrm{StGB}$ ). Bremer Forschungen zur Kriminalpolitik, Bd. 10. LIT, Berlin

Querengässer J, Baur A (2021) Prädiktoren des Entlassmodus aus forensischer Suchtbehandlung - Erster Teil einer Übersichtsarbeit zu Erfolgsdeterminanten einer Unterbringung gemäß § 64 StGB. Fortschr Neurol Psychiatr. https://doi.org/10.1055/a-1389-5782

Querengässer J, Hoffmann K, Ross T (2014) Die Meinung der Anderen - Wie die Haltung wichtiger Bezugspersonen den Verlauf einer Entziehungsmaßregel beeinflusst. https://www.researchgate. net/publication/284731341. Zugegriffen: 3. Mai 2021

Rauchfleisch U (1991) Psychodynamik und Psychotherapie von Alkoholabhängigen mit dissozialen Tendenzen. Sucht 37:289-299

Rauchfleisch U (1999) Außenseiter der Gesellschaft. Psychodynamik und Möglichkeiten zur Psychotherapie Straffälliger. Vandenhoeck \& Ruprecht, Göttingen

Schalast N (2000) Therapiemotivation im Maßregelvollzug gemäß $\S$ 64 StGB. Fink, München

Schalast N (2019) Ergebnisse der Essener Evaluationsstudie. In: Schalast N (Hrsg) Straffällige mit Suchtproblemen. Pabst, Lengerich, S $29-150$

Schalast N (2021) Zur Debatte um die Reform der gesetzlichen Voraussetzungen Unterbringung in der Entziehungsanstalt. Forens Psychiatr Psychol Kriminol. https://doi.org/10.1007/s11757-02100652-1

Schalast N, Frey M, Boateng S, Dönisch-Seidel U, Leygraf N (2019) Was rechtfertigt eine Behandlungsmaßregel für Täter mit Suchtproblemen? Recht Psychiatr 37(2021):141-146. https://doi.org/ 10.1486/RP-2019-03_141

Seifert D, Leygraf N (1999) Drogenabhängige Straftäter im Maßregelvollzug Ergebnisse einer Querschnittserhebung. Nervenarzt 70(5):450-456

Wilson D, Sperber D (2012) Meaning and relevance. University Press, Cambridge 